Research Paper

\title{
An experimental and analytical study on the feasibility of SMA spring driven actuation of an iris mechanism
}

\author{
Aravindh Rajan ${ }^{a}$, Mostafa Abouseada a ${ }^{a}$ Pavithra Manghaipathy ${ }^{\mathrm{a}}$, Nesrin Ozalp ${ }^{\mathrm{b}, *}$, Feras Abdul Majid ${ }^{\mathrm{a}}$, \\ Ayman Salem ${ }^{a}$, Arun Srinivasa ${ }^{c}$ \\ a Texas AEM University at Qatar, Mechanical Engineering Department, P.O. Box 23874, Doha, Qatar \\ ${ }^{\mathrm{b}} \mathrm{KU}$ Leuven, Mechanical Engineering Department, 3001 Leuven, Belgium \\ ${ }^{\mathrm{c}}$ Texas AEM University, Mechanical Engineering Department, College Station, TX 77843, USA
}

\section{H I G H L I G H T S}

- An iris mechanism proposed in response to fluctuations in solar energy.

- Iris motion controlled with the use of Nickel-Titanium Shape Memory Alloy springs.

- Variation of the force exerted by SMA spring w.r.t. temperature tested experimentally.

- An analytical expression that relates the aperture area and crank rotation is given.

- SMA springs showed promising actuation mechanism as a replacement to motor.

\section{A R T I C L E I N F O}

Article history:

Received 24 December 2015

Accepted 8 April 2016

Available online $\mathrm{xxxx}$

\section{Keywords:}

Iris mechanism

Aperture

Solar energy

Shape Memory Alloys (SMA) spring

Analytical calculations

\begin{abstract}
A B S T R A C T
Variation in incoming solar energy adversely affects the temperature inside a solar reactor and lowers its efficiency. Therefore, it is important to develop a mechanism that can maintain semi-constant temperatures inside the reactor from sunrise to sunset. In this paper, we present an iris mechanism that reduces or enlarges its circular opening with the use of Nickel-Titanium Shape Memory Alloy (SMA) springs. SMA springs possess memory of their shapes at certain temperatures. Hence, by controlling the temperature of the spring, it is possible to exert different forces that may then be transferred to the variable aperture mechanism. In this study, variation of the force exerted by an SMA spring with respect to temperature was experimentally tested and the viability of an SMA spring's use in actuating an iris mechanism aperture was examined. In order to simulate conditions under fluctuating solar radiation, a $7 \mathrm{~kW}$ solar simulator was used in experiments at varying power levels. It was observed that SMA springs are promising as a replacement of the actuation mechanism driven by a motor.
\end{abstract}

(c) 2016 Elsevier Ltd. All rights reserved.

\section{Introduction}

Persistent emission of greenhouse gases from various sectors and depletion of hydrocarbon resources due to heavy reliance on fossil fuel conversion technologies have led to serious considerations to accommodate more renewable sources in power generation and fuel production. Therefore, focus on solar energy research is now being on the rise for testing various thermochemical processes, such as methane splitting, thermo-catalytic decomposition of $\mathrm{CH}_{4}, \mathrm{CO}_{2}$ capture from air via carbonation, calcination cycles. The current set up for a directly irradiated solar reactor

\footnotetext{
* Corresponding author.

E-mail address: nesrin.ozalp@kuleuven.be (N. Ozalp).
}

generally consists of an aperture or a window to absorb the solar radiation and a reactor body that varies according to the process and design constraints. A key concept of a solar reactor is to maintain a constant temperature inside the reactor's cavity in order to sustain a uniform temperature distribution for highest efficiency possible [1].

The fluctuation of solar radiation due to the position of the sun, unavailability during night time, and transient availability at various weather conditions is the main challenge faced when designing a solar reactor and accompanying auxiliary systems. This is due to the fact that a solar reactor needs to achieve uniform temperature distribution and uninterrupted use of process heat in the reactor's cavity to maximize its efficiency. Currently, there are few techniques that are being used to accommodate the transient periods and control the reactor's temperature. One of the commonly 


\section{Nomenclature}

$\begin{array}{ll}M_{S} & \text { martensite start temperature } \\ P & \text { \% of platinum } \\ A_{s} & \text { austenite start temperature } \\ A_{f} & \text { austenite finish temperature }\end{array}$

$T \quad$ torque produced by bias spring

$\tau_{s} \quad$ torque produced by SMA spring practiced methods is to adjust the mass flow rate of the feedstock which is very simple to implement.

Another method is the focusing and defocusing of the heliostats, which requires careful control of the heliostat field. For example, Säck et al. developed a system to control separate groups of heliostats to focus the radiation on the receiver module [2]. Each group of heliostats was given a specific control and by changing the heliostat's angle; it was possible to maintain a constant cavity temperature for the two-step water thermochemical cycle. In addition, another technique involving heliostats is to change the layouts of the heliostat field to improve optical efficiency. For example, Ref. [3] proposed a model for heliostat optimization which shows that the most efficient layout for the heliostat field depends on a lot of factors such as location of latitude, altitude, and cloud coverage.

Finally, thermal storage is also being widely used to ensure continuous operation. The underlying idea of thermal energy storage is to capture energy during the peak times and reuse that during the transient times. Additionally, this thermal energy can be used as a substitute for combustion processes or could also store the energy expelled during an exothermic reaction. Thermal energy storage systems can be categorized as follows: (1) solid media (2) molten salts, and (3) Phase Change Materials (PCMs) on basis of global impact per kWh stored [4]. Although solid media storage system is the most environment friendly method, it offers a low energy storage capacity. On the other hand, the molten salts storage system has the highest environmental impact which is basically the most commonly used method. Ideally, the desired choice would be PCMs due to higher energy storage density over smaller temperature ranges which would in turn reduce the size of the storage unit for a latent heat storage. However, the main problem with PCMs is the solidification on the heat transfer system due to low thermal conductivity.

Although these techniques are widely being practiced, there are several drawbacks associated with each of them. For example, although the temperature inside a solar reactor can be easily controlled by varying the mass flow rate of the feedstock, it disturbs the flow dynamics inside the reactor [4]. This is a major problem for cases where the flow pattern must be maintained constant. For example, in solar thermal cracking of natural gas; carbon particles deposit on optical window [5], and clog the reactor exit [6]. To reduce carbon deposition on reactor walls and at the exit, one promising approach is to maintain special swirling flow pattern inside the reactor [7]. Therefore, temperature inside the reactor should be controlled without changing the flow rate so that the flow pattern remains undisturbed. In order to achieve that, a special mechanism or a design is required.

In this paper, we present a novel approach with the use of Shape Memory Alloy (SMA) springs as a promising method to actuate an iris mechanism for the maintenance of semi-constant temperature inside a solar reactor without disturbing the flow dynamics and without using any motor controlled mechanisms or apparatuses.

\section{Current state of the art}

Current solar reactor designs have a fixed aperture size which does not compensate for the changes in variation in solar energy from the sunrise to sunset. On the other hand, by varying the aperture radius with incoming flux as proposed in Ref. [8], temperature inside the reactor would be kept semi-constant in theory per heat transfer and optical analysis [9]. Such mechanism has the potential to stabilize internal reactor operating conditions by minimizing radiation losses through the aperture and respond to the fluctuations in solar flux by regulating the aperture area. However, it is a challenge to actuate the blades of the iris mechanism with a motor under such high temperatures even if the iris is water cooled and the motor is air cooled. Therefore, an alternative mechanism is needed to avoid running control system tools under high temperatures. SMA springs are very promising to be considered as a viable solution. They are not being affected by the high temperature or thermal stress due to temperature changes via flux changes. In fact, they make use of the temperature difference and can withstand very high temperatures. SMA springs can respond to temperature changes due to changes in direct normal radiation and can actuate the iris mechanism blades to obtain the appropriate aperture size. It is important to select the correct SMA spring and size that would yield accurate aperture size opening and closure. The optimum aperture size depends on the magnitude of direct normal insolation.

For example, if we refer to a typical daily cycle of direct normal radiation (I) from the sun recorded by the National Renewable Energy Laboratory (NREL) database [10], the normal insolation can be calculated based on the clear sky model by Bird and Hulstrom [11]. The direct irradiance reaches a peak of approximately $981 \mathrm{~W} / \mathrm{m}^{2}$ at $12: 31$ whereas its minimum is $200 \mathrm{~W} / \mathrm{m}^{2}$ at $04: 49$ and 19:21 [8]. The power intercepted by the aperture as a function of its diameter can be calculated using the mean concentration ratio of the paraboloidal concentrator and the normal beam insolation as seen in Fig. 1.

Referring to Fig. 1, an aperture size of $4 \mathrm{~cm}$ intercepts a maximum of about $5.5 \mathrm{~kW}$ for a peak normal beam insolation of $981 \mathrm{~W} / \mathrm{m}^{2}$ at $12: 31$. For the same aperture size, this reduces to $1.12 \mathrm{~kW}$ for normal insolation of $200 \mathrm{~W} / \mathrm{m}^{2}$ in the morning at 04:49 and evening at 19:21. In order to compensate for this dramatic change in power intercepted from morning to evening, a variable aperture would be used where the optimum size is set according to the incoming power level.

Depending on the magnitude of direct normal insolation, there is an optimum aperture size that can maintain the desired level of temperature inside the reactor for a particular time of the day. However, it should be noted that although larger aperture size implies higher $P_{a p}$, this may not necessarily mean higher temperature because a larger aperture size causes higher re-radiation losses back through the aperture. Therefore, selecting an optimum aperture size for a particular time of the day is a compromise between maximizing radiation captures and minimizing reradiation losses [12].

Although a semi-constant temperature would be conceptually maintained using variable aperture mechanism; design, manufacturing, and control of a variable aperture mechanism suitable for solar thermochemical reactors are not available in literature. However, a variety of iris-type mechanisms for various other applications can be found as seen in Table 1. There are many different aperture designs, each having different components, structure, 


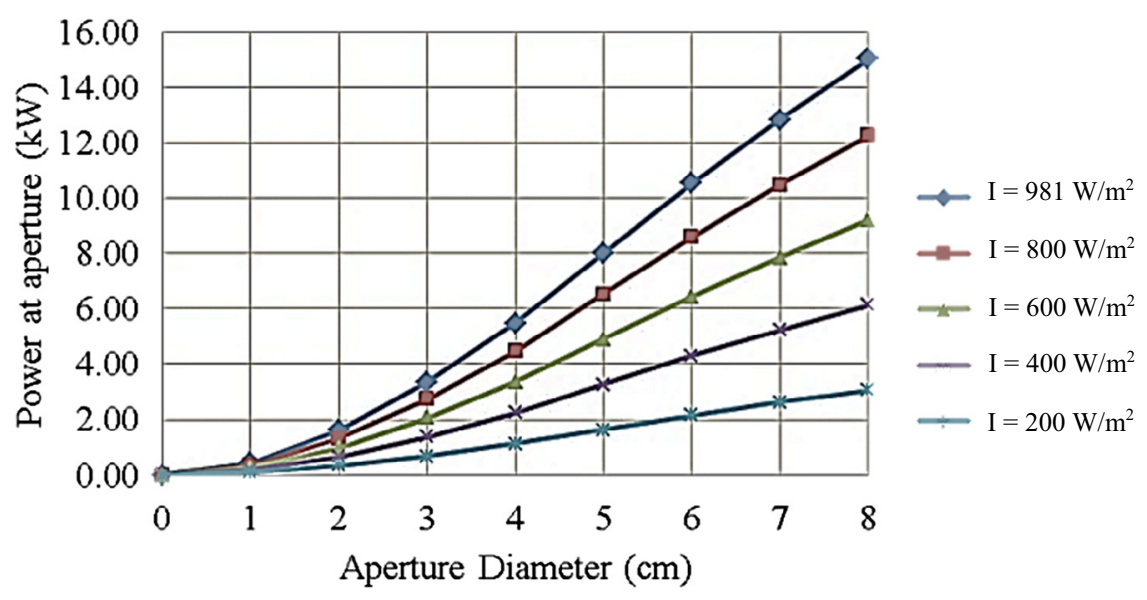

Fig. 1. Power intercepted at aperture as a function of diameter for different levels of normal insolation [8].

Table 1

A comparison of variable aperture (iris mechanism) designs.

\begin{tabular}{|c|c|c|c|c|c|c|}
\hline Reference & [13] & {$[14]$} & [15] & [16] & {$[17]$} & [18] \\
\hline Field of application & Imaging systems & Optics & Radiotherapy & $\begin{array}{l}\text { Energy beam } \\
\text { collimators }\end{array}$ & Lens technology & Robotic radiosurgery \\
\hline Media flow & Visible light & Visible light & Radiation beams & $\begin{array}{l}\text { Energy beams } \\
\text { (radiation) }\end{array}$ & Light & $\mathrm{X}$-rays \\
\hline Control mechanism & $\begin{array}{l}\text { Manually by a } \\
\text { handle }\end{array}$ & Separate linear actuators & $\begin{array}{l}\text { Rotating driving } \\
\text { plates }\end{array}$ & $\begin{array}{l}\text { Rotatable shafts and } \\
\text { actuators }\end{array}$ & $\begin{array}{l}\text { Power driver and } \\
\text { rotatable ring }\end{array}$ & $\begin{array}{l}\text { Drive motor and cam } \\
\text { plate }\end{array}$ \\
\hline Aperture structure & $\begin{array}{l}5 \text { Curved sliding } \\
\text { blades }\end{array}$ & 4 Shutter blades & 6 Trapezoidal blocks & 4 Blocks & $\begin{array}{l}\text { Multiple curved } \\
\text { blades }\end{array}$ & $\begin{array}{l}\text { Twelve prism- shaped } \\
\text { segments }\end{array}$ \\
\hline Component motion & Rotational & Sliding & Sliding & Rotational & Rotational & Translational \\
\hline $\begin{array}{r}\text { Component } \\
\text { material }\end{array}$ & Metal & $\begin{array}{l}\text { Bonded silicon-on- } \\
\text { insulator material }\end{array}$ & Brass & Opaque material & Metal or plastic & $\begin{array}{l}\text { Tungsten - copper } \\
\text { alloy }\end{array}$ \\
\hline $\begin{array}{l}\text { Cross-sectional } \\
\text { exposure }\end{array}$ & Circle & Square & Hexagon & Square & Circle & Approximately circle \\
\hline $\begin{array}{l}\text { Schematic of design } \\
\text { concept }\end{array}$ & & & & & & \\
\hline
\end{tabular}

control mechanism, and field of application. The designs vary from having four blades or segments to having twelve blades in the case illustrated in Ref. [18], or more as illustrated in Ref. [17]. The most effective aperture designs with simple aperture mechanisms will be those having a circular aperture area.

By considering multiple blade arrangements previously studied, it can be seen that by increasing the number of blades of a solar aperture, the system becomes closer to an iris mechanism. Therefore, increasing the number of blades produces a more circular aperture area. Hence, reaching an aperture area of an exact circle at infinity number of blades. The design proposed by the authors took advantage of this behavior, where it was constructed with eight blades to reach a fine approximate of a circle. However, not having too much blades in order to avoid any difficulties in the control mechanism. In the earlier studies conducted by the authors, a simple variable aperture design mechanism was produced, consisting of only two blades. Then a more sophisticated aperture mechanism was developed to obtain a more circular aperture than the older design which is shown in Fig. 2. This four-bar crank-rocker mechanism is employed to open/close the aperture. The position and dimensions of the linkages are configured in such a way that the system behaves as a crank-rocker mechanism. The disk fixed to the motor (crank) is free to rotate. The top plate of the aperture (rocker) is connected to the crank via a connecting link that oscillates between two set points defined as the closing (totally closed aperture) and opening (totally opened aperture) angles of the aperture. In this way, no matter how many revolutions the crank plate makes, the aperture position would toggle from completely opened to completely closed position, and vice versa. The geometric dimensions for the different parts of this design are shown in Fig. 3.

The operation of the mechanism is governed by a motor attached to the center of the plate that provides the crank link. The crank turns along with the motor and in turn, rotates the rocker. This is due to the mechanical constraint provided by the coupler. The eight blades are pivoted on a fixed base plate with each blade separated by an arc length, along the circumference, as subtended by a $45^{\circ}$ angle at the base plate center. This leads to an octagon shape for the aperture as viewed perpendicular to the top plate plane which is illustrated in Fig. 4. The area of the octagon depends on the amount of rotation experienced by the rocker. This way, the most optimum area for a certain flux distribution may be obtained.

\section{Calculation of variation in aperture area and identification of corresponding aperture rotation}

The first step of establishing a relationship between the SMA spring actuation and the aperture mechanism is to analyze the 

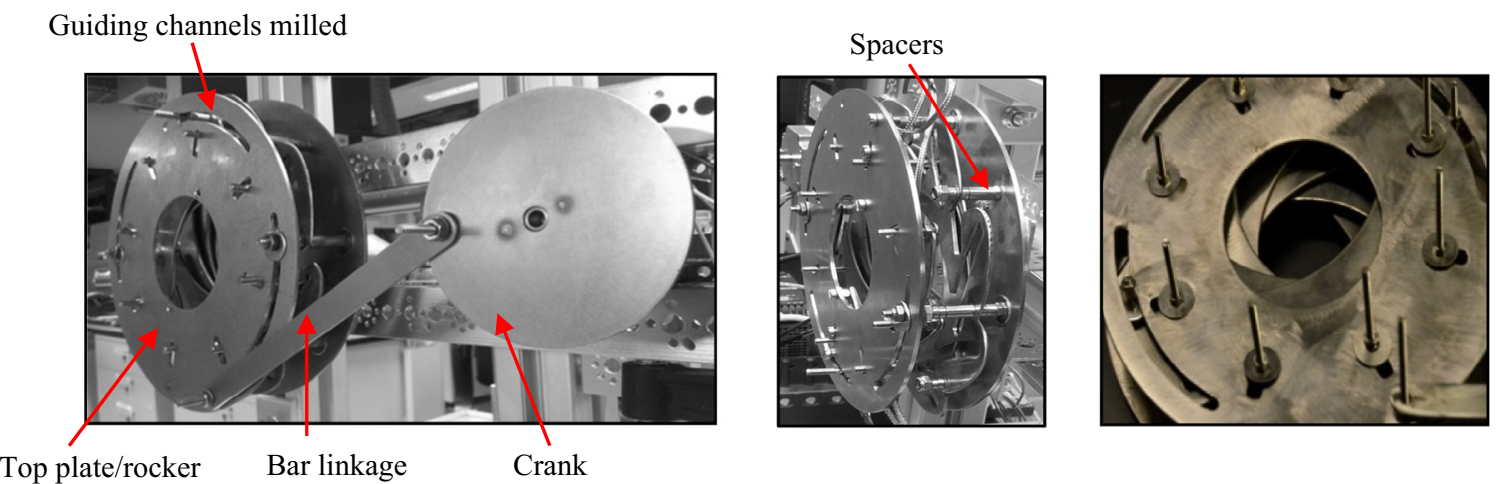

Fig. 2. Variable aperture mechanism developed at the Sustainable Energy Research Laboratory (SERL) [19].

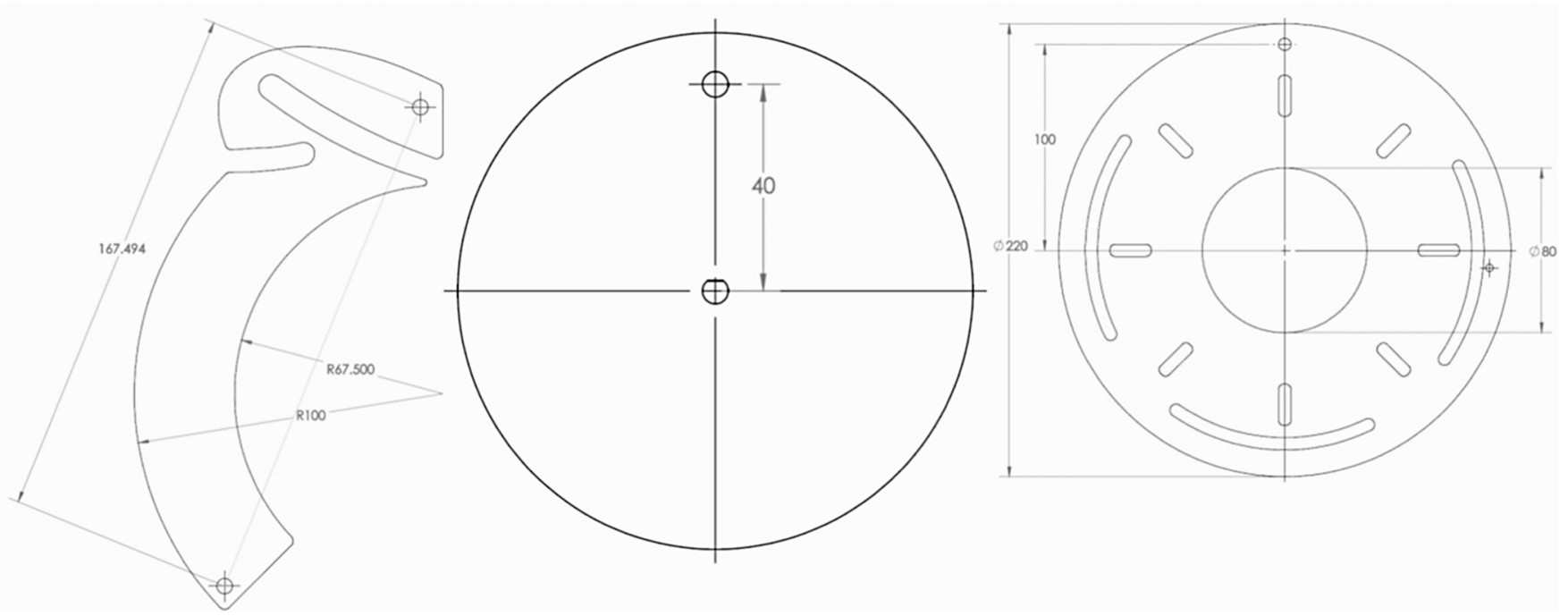

Fig. 3. Geometric dimensions for different parts of the design. Blade on the left, motor plate (crank) in the center, and the aperture top plate (output) on the right.
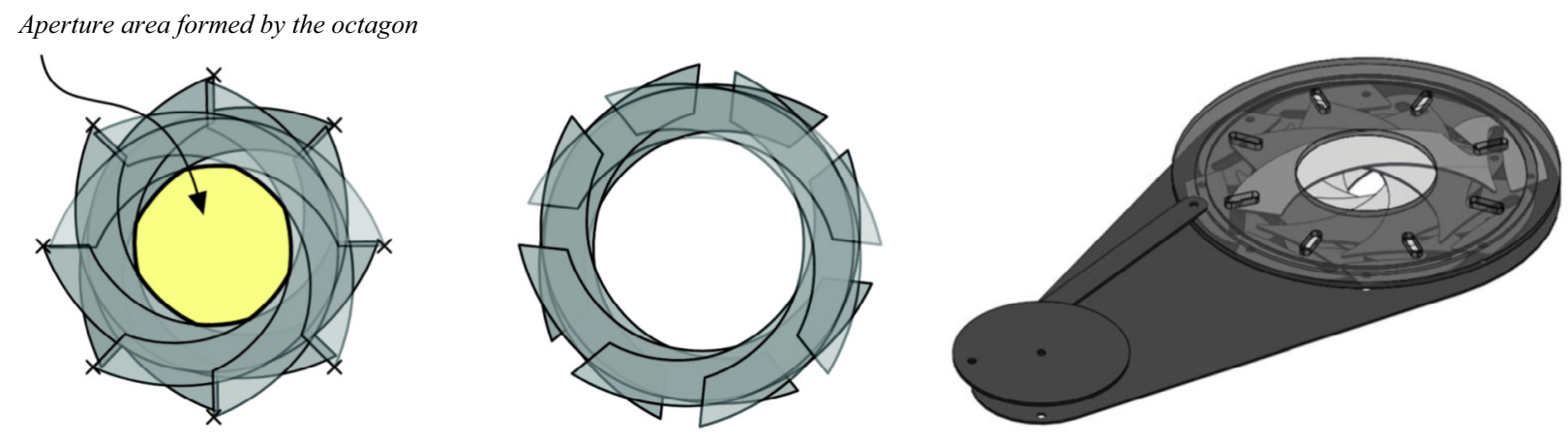

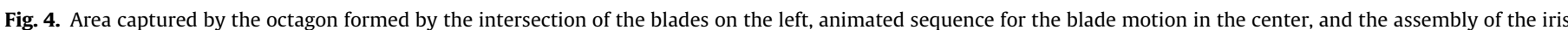
mechanism on the right.

aperture mechanism and develop an analytical expression that relates the aperture area and crank rotation. For this, the variable aperture shown in Fig. 2 is modeled as a Grashof crank-rocker mechanism as shown in Fig. 5, where the position vectors for the four links are indicated. The advantage of having a Grashof crank-rocker mechanism and the input being the crank, is that toggle positions can be avoided with the smallest link making a complete revolution (the input link in this case).

In order to analyze the position of the four-bar mechanism, the position vector loop for the mechanism and local-non-rotating coordinates for each link should be demonstrated as seen in Fig. 6 . The position vectors for the four links are shown in Fig. 6.

The lengths $a, b, c$ and $d$ correspond to the crank (input), coupler, rocker (output) and ground link respectively. In order to control the variable aperture, it is necessary to relate the aperture top plate rotation angle $\theta_{4}$ to the input angle $\theta_{2}$. Once the mathematical relationship between $\theta_{4}$ and $\theta_{2}$ is established, this information can be extended to calculate the corresponding aperture area as a function of $\theta_{4}$. A brief outline of this derivation is provided below. The lengths labeled as $a, b, c$ and $d$ are known constants 


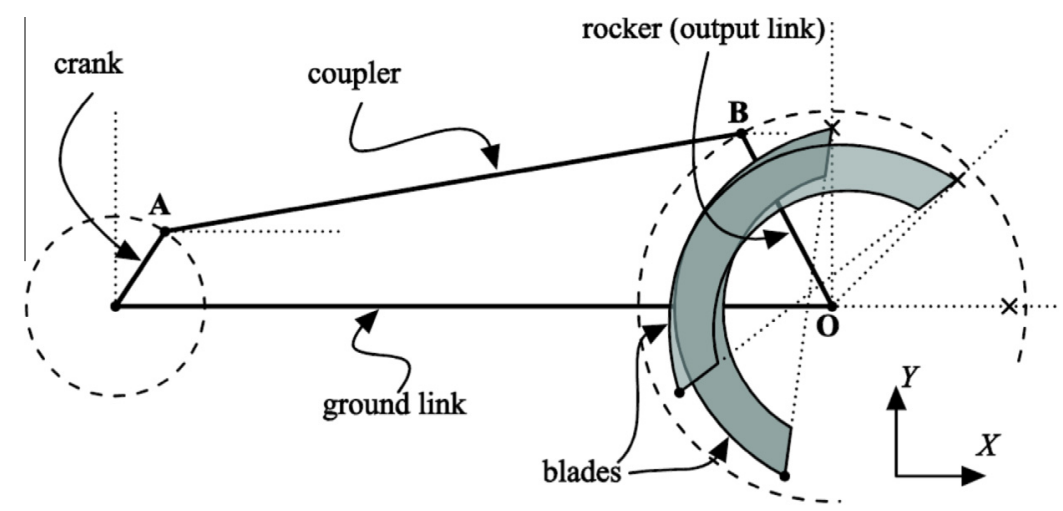

Fig. 5. Four bar crank-rocker mechanism with the aperture blades [19].

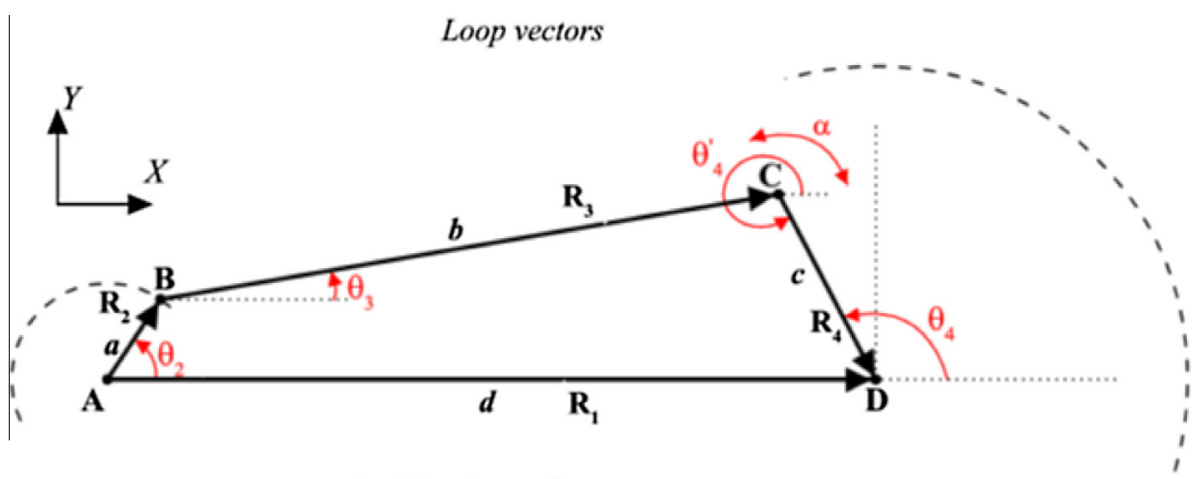

Link local coordinate sytems

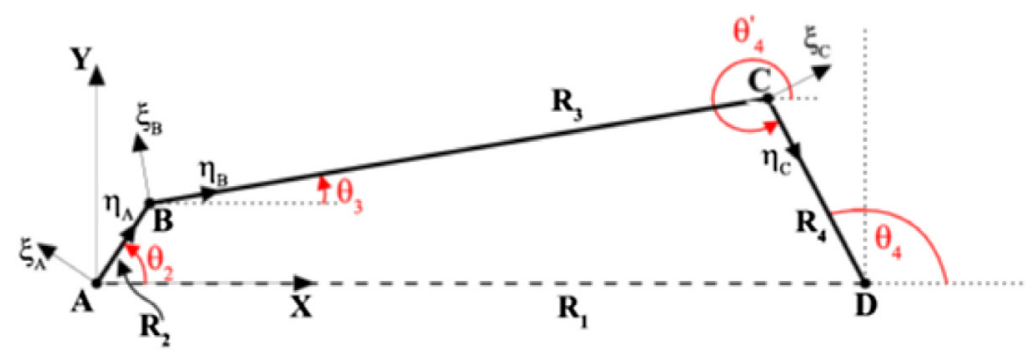

Fig. 6. Position vector loop for the four-bar mechanism and local-non-rotating coordinates for each link.

and the angle made by the ground link with the horizontal, $\theta_{1}=0$. An implicit relation between $\theta_{2}$ and $\theta_{4}$ can be found as follows:

From the vector loop $\overrightarrow{A B C D A}$ in Fig. 6, we notice that $\vec{a}+\vec{b}+\vec{c}+\vec{d}=0$. Resolving the vector into the $x-y$ co-ordinates gives that $\left(a \cos \theta_{2} \boldsymbol{i}+a \sin \theta_{2} \boldsymbol{j}\right)+\left(b \cos \theta_{3} \boldsymbol{i}+b \sin \theta_{3} \boldsymbol{j}\right)$ $+\left(-c \cos \theta_{4} \boldsymbol{i}-c \sin \theta_{4} \boldsymbol{j}\right)-d \boldsymbol{i}=0$. This implies that the summation of the components of the vectors along the $x$ and $y$ axes is zero separately.

Pairing the $x$ and $y$ components,

$a \cos \theta_{2}+b \cos \theta_{3}-c \cos \theta_{4}-d=0$

$a \sin \theta_{2}+b \sin \theta_{3}-c \sin \theta_{4}=0$

From Eqs. (1) and (2) respectively,

$\cos \theta_{3}=\frac{d+c \cos \theta_{4}-a \cos \theta_{2}}{b}$

$\sin \theta_{3}=\frac{c \cos \theta_{4}-a \sin \theta_{2}}{b}$
Using Eqs. (3) and (4),

$$
\begin{aligned}
\cos ^{2} \theta_{3}+\sin ^{2} \theta_{3}= & \left(\frac{d+c \cos \theta_{4}-a \cos \theta_{2}}{b}\right)^{2} \\
& +\left(\frac{c \cos \theta_{4}-a \sin \theta_{2}}{b}\right)^{2}=1
\end{aligned}
$$

Expanding Eq. (5),

$$
b^{2}=c^{2}+d^{2}+a^{2}+2 c d \cos \theta_{4}-2 a d \cos \theta_{4}
$$

$$
-2 a c\left(\cos \theta_{4} \cos \theta_{2}+\sin \theta_{4} \sin \theta_{2}\right)
$$

Rearranging Eq. (6),

$\cos \left(\theta_{4}-\theta_{2}\right)=\frac{a^{2}-b^{2}+c^{2}+d^{2}}{2 a c}+\frac{d}{a} \cos \theta_{4}-\frac{d}{c} \cos \theta_{2}$

Let $K_{1}=d / a, K_{2}=d / c$ and $K_{3}=a^{2}-b^{2}+c^{2}+d^{2} / 2 a c$. Eq. (7) then becomes,

$\cos \left(\theta_{4}-\theta_{2}\right)=K_{3}+K_{1} \cos \theta_{4}-K_{1} \cos \theta_{2}$

By using the following identities, 
$\sin \theta_{4}=\frac{2 \tan \frac{\theta_{4}}{2}}{1+\tan ^{2} \frac{\theta_{4}}{2}} \quad$ and $\quad \sin \theta_{4}=\frac{2 \tan \frac{\theta_{4}}{2}}{1+\tan ^{2} \frac{\theta_{4}}{2}}$

Eq. (8) becomes,

$A \tan ^{2}\left(\frac{\theta_{4}}{2}\right)+B \tan \left(\frac{\theta_{4}}{2}\right)+C=0$

where $A=K_{3}-K_{1}+\cos \theta_{2}\left(1-K_{2}\right), \quad$ and; $B=-2 \sin \theta_{2}, \quad$ and $C=K_{1}+K_{3}-\cos \theta_{2}\left(K_{2}+1\right)$.

The solution of quadratic Eq. (9) gives the following desired mathematical relationship between $\theta_{4}$ and $\theta_{2}$.

$\tan \frac{\theta_{4}}{2}=\frac{-B \pm \sqrt{B^{2}-4 A C}}{2 A}$

Fig. 7 shows the variation of $\theta_{4}$ with $\theta_{2}$ for varying lengths of ground linkage ' $d$ ' obtained using Eq. (10). The lowest plot corresponds to $d=200 \mathrm{~mm}$, and the top most plot corresponds to $d=260 \mathrm{~mm}$. As the value of $d$ increases from $200 \mathrm{~mm}$ to $260 \mathrm{~mm}$, it is observed that the change in $\theta_{4}$ is greater. This allows for a greater change in area, which is desirable for the aperture mechanism.

From the Grashof condition, $d<260 \mathrm{~mm}$, and in order to facilitate a smoother transition, $250 \mathrm{~mm}$ was chosen as the optimum value of the ground linkage. Fig. 8 shows the variation of $\theta_{4}$ with $\theta_{2}$ exclusively for $d=250 \mathrm{~mm}$.

The blades are pivoted at $E_{i}$ at equal intervals. Therefore, the first blade is $45^{\circ}$ from the positive, the second is at $90^{\circ}$ and so forth. Fig. 9 shows the first blade that is fixed on to the base plate. All blades undergo a rotation of $\beta$ as shown in Fig. 9. The value of $\beta$ for all blades is the same, owing to the geometry.

The angle $\theta_{c}$ formed by the rocker and $O E_{i}$ is a geometrically constant at all times and its value is $89.61^{\circ}$. In triangle $\widehat{\mathrm{A}_{l} \mathrm{OE}}$, the exterior angle theorem yields:

$\frac{O A_{i}}{\sin \gamma}=\frac{A_{i} E_{i}}{\sin \widehat{A}_{l} \widehat{O E}_{l}}$

$\frac{r_{i}}{\sin \gamma}=\frac{e}{\sin \left(\theta_{c}+\theta_{4}-\theta_{i}\right)}$

$\gamma=\arcsin \left[\frac{r_{i}}{e} \sin \left(\theta_{c}+\theta_{4}-\theta_{i}\right)\right]$

$\beta=\gamma+\theta_{4}-\theta_{i}+\theta_{C}$

The next step is the calculation of the aperture area. Area $A$ of the regular octagon shown in Fig. 10, with sides of length $a$, is given as follows:

$A_{o c t}=2 a^{2}(1+\sqrt{2})$

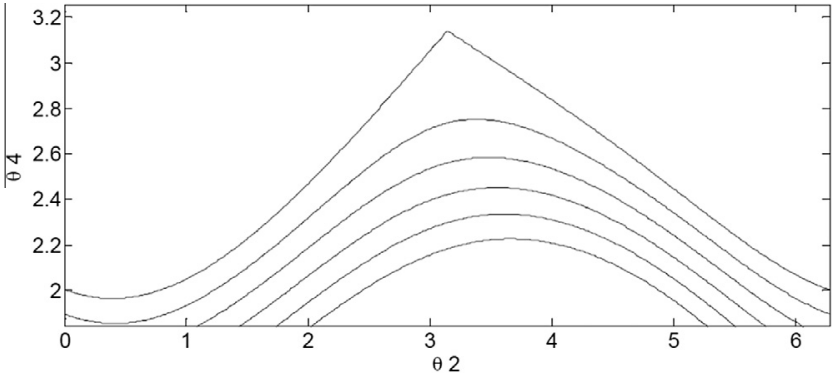

Fig. 7. Variation of $\theta_{4}$ with $\theta_{2}$ for varying ground linkage lengths.

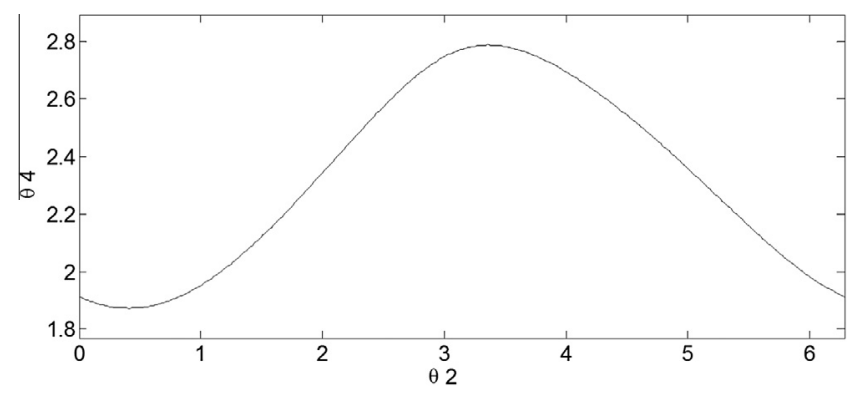

Fig. 8. Variation of $\theta_{4}$ with $\theta_{2}$ for varying $d=250 \mathrm{~mm}$.

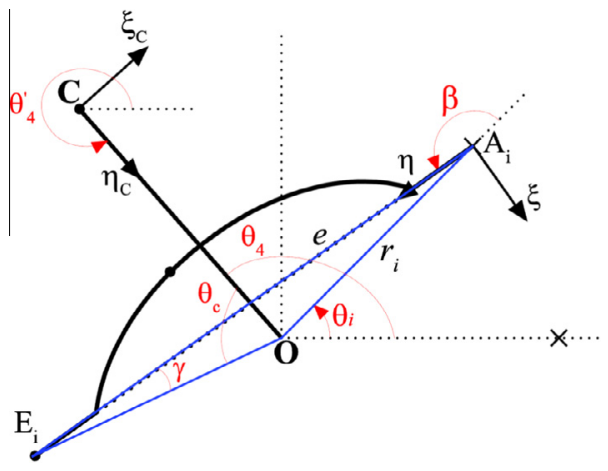

Fig. 9. Positioning of the first blade and related angles.

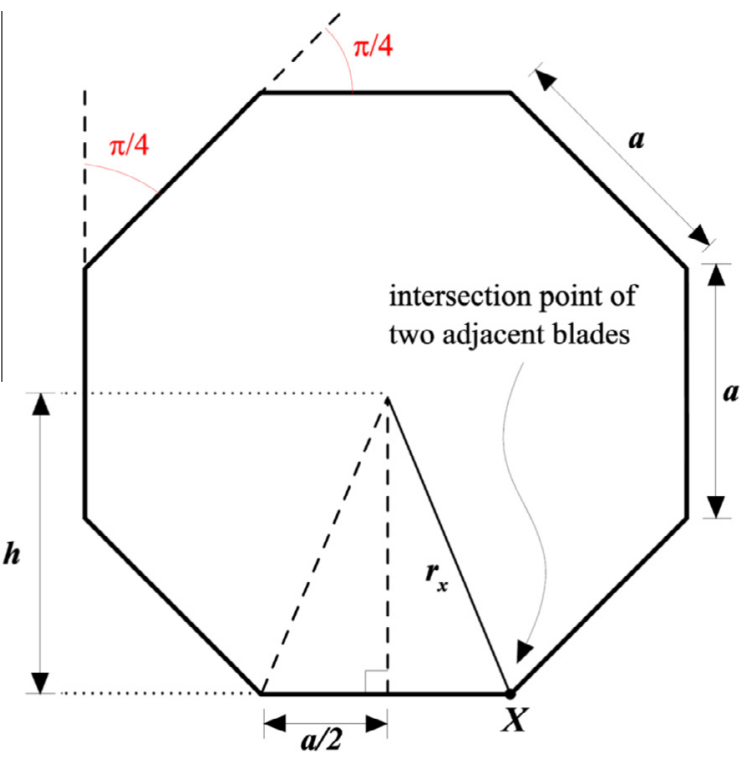

Fig. 10. Regular octagon with sides of length $a$.

The length $a$ can then be related to length $r_{X}$, which is the distance from the center of the octagon to one of its vertices. This point is of most interest, since any two adjacent blades' inner edge intersect at $X$.

$h=\left(\frac{a}{2}+\frac{a}{\sqrt{2}}\right)$

$r_{X}^{2}=\left(\frac{a}{2}\right)^{2}+h^{2}$

$a=\frac{r_{X}}{\sqrt{1+\frac{1}{\sqrt{2}}}} p$ 
$A_{o c t}=f\left(r_{X}\right)$

Eqs. (18) and (19) imply that if the direction vector $R_{X}$ (point $O$ in Fig. 5 to point $X$ ) is known, then the area of the aperture can be determined.

The local-rotating-coordinate system, $\eta_{i}-\xi_{i}$, for blade $i$, where $i=1,2,3 \ldots, 8$ pivoted at point $A_{i}$ is shown in Fig. 11 below. The $\eta-\xi$ coordinate system is located at the pivot point and the rotation angle $\beta$ depends on the blade rotation about $A_{i} . x_{i}-y_{i}$ is the local-non-rotating-coordinate system for the $i^{\text {th }}$ blade related by an angle $\theta_{i}$ to the global $x-y$ coordinate system. Angle $\psi$ is the angular shift between two adjacent blades, this is a constant with a value equal to $\pi / 4$.

The local coordinate systems are related to the global coordinate system by the following combination of rotation and translation matrices:

$$
\begin{aligned}
& {\left[\begin{array}{l}
x_{i} \\
y_{i}
\end{array}\right]=\left[R_{1}\right]\left[\begin{array}{l}
x \\
y
\end{array}\right]} \\
& {\left[\begin{array}{l}
x_{i+1} \\
y_{i+1}
\end{array}\right]=\left[R_{2}\right]\left[\begin{array}{l}
x_{i} \\
y_{i}
\end{array}\right]} \\
& {\left[\begin{array}{l}
\eta_{i} \\
\xi_{i}
\end{array}\right]=\left[R_{3}\right]\left[\begin{array}{l}
x_{i} \\
y_{i}
\end{array}\right]} \\
& {\left[\begin{array}{l}
\eta_{i+1} \\
\xi_{i+1}
\end{array}\right]=\left[R_{3}\right]\left[\begin{array}{l}
x_{i+1} \\
y_{i+1}
\end{array}\right]}
\end{aligned}
$$

Position vector with respect to (w.r.t.) $x-y(i, j)$ for any point along the inner edge of blade $i$ pivoted at $A_{i}$ is:

$$
\left[\begin{array}{l}
x \\
y
\end{array}\right]=\left[T_{1}\right]+\left[R_{1}\right]^{T}\left[R_{3}\right]^{T}\left[\begin{array}{l}
\eta_{i} \\
\xi_{i}
\end{array}\right]
$$

Position vector w.r.t. $x-y(i, y)$ for any point along the inner edge of the $(i+1)^{\text {th }}$ blade pivoted at $A_{i+1}$ is:

$$
\left[\begin{array}{l}
x \\
y
\end{array}\right]=\left[T_{2}\right]+\left[R_{1}\right]^{T}\left[R_{2}\right]^{T}\left[R_{3}\right]^{T}\left[\begin{array}{l}
\eta_{i+1} \\
\xi_{i+1}
\end{array}\right]
$$

where

$$
\begin{aligned}
& R_{1}=\left[\begin{array}{cc}
\cos \theta_{i} & \sin \theta_{i} \\
-\sin \theta_{i} & \cos \theta_{i}
\end{array}\right] ; \quad R_{2}=\left[\begin{array}{cc}
\cos \psi & \sin \psi \\
-\sin \psi & \cos \psi
\end{array}\right] ; \\
& R_{3}=\left[\begin{array}{cc}
\cos \beta & \sin \beta \\
-\sin \beta & \cos \beta
\end{array}\right] ; \quad\left[T_{1}\right]=\left[\begin{array}{l}
x_{A i} \\
y_{A i}
\end{array}\right] ; \quad\left[T_{2}\right]=\left[\begin{array}{l}
x_{A i+1} \\
y_{A i+1}
\end{array}\right]
\end{aligned}
$$

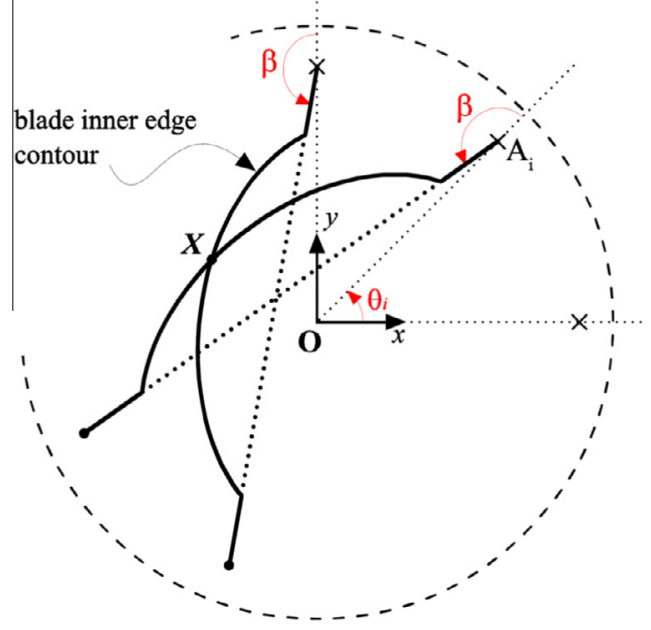

At the point of intersection, $X$, Eq. (24)=Eq. (25). This gives:

$$
\left[T_{1}\right]+\left[R_{1}\right]^{T}\left[R_{3}\right]^{T}\left[\begin{array}{l}
\eta_{i} \\
\xi_{i}
\end{array}\right]=\left[T_{2}\right]+\left[R_{1}\right]^{T}\left[R_{2}\right]^{T}\left[R_{3}\right]^{T}\left[\begin{array}{l}
\eta_{i+1} \\
\xi_{i+1}
\end{array}\right]
$$

Since $\xi_{i}=\left(\eta_{i}\right)$ and $\xi_{i}+1=\left(\eta_{i}+1\right)$, in the above system of equations there are two unknowns, namely $\eta_{i}$ and $\eta_{i}+1$.

$\xi_{i}=\xi_{c}-\sqrt{r_{c}^{2}-\left(\eta_{i}-\eta_{c}\right)^{2}}$

$\xi_{i+1}=\xi_{c}-\sqrt{r_{c}^{2}-\left(\eta_{i+1}-\eta_{c}\right)^{2}}$

where $\xi_{c}$ and $\eta_{c}$ are known constants.

Eq. (26) is an implicit equation with two variables. The solution for the nonlinear system is found using MATLAB's fsolve function available as part of the optimization toolbox. Once $\eta_{i}$ and $\eta_{i+1}$ are known, the coordinates of point $X$ can be calculated using either Eq. (24) or (25). The variation of the aperture's area with respect to $\theta_{2}$ is demonstrated below in Fig. 12 .

\section{Selection of SMA spring}

During the design of a variable aperture mechanism, particular attention needs to be given to the materials selection, ease of manufacturing, and the actuation system that can work for that design. As demonstrated earlier, one way of actuating an iris mechanism aperture can be accomplished by using a planetary gear system and four-bar mechanical linkage. However, since the operating temperature of solar reactors is very high, this technique cannot be used as it has several disadvantages at high temperatures such as the use of stepper motors which need to be cooled constantly. However, SMAs can be used to overcome these disadvantages along with their challenges in design and manufacturing. For example, with the use of SMA springs, it is possible to control the opening of this aperture mechanism by changes in the amount of incoming energy.

SMAs are a special group of intermetallic alloys. The most well-known of them being the almost equiatomic combination of Nickel and Titanium, which goes under the trade name of Nitinol. They have the unique property of undergoing a reversible solid to solid phase transition from a low temperature tetragonal phase to a high temperature cubic phase. Technological interest in these alloys originates from: (1) their extremely high strength (above $900 \mathrm{MPa}$ ) and their insensitivity to environmental conditions, (2) their ability to change their configuration and shape with changes

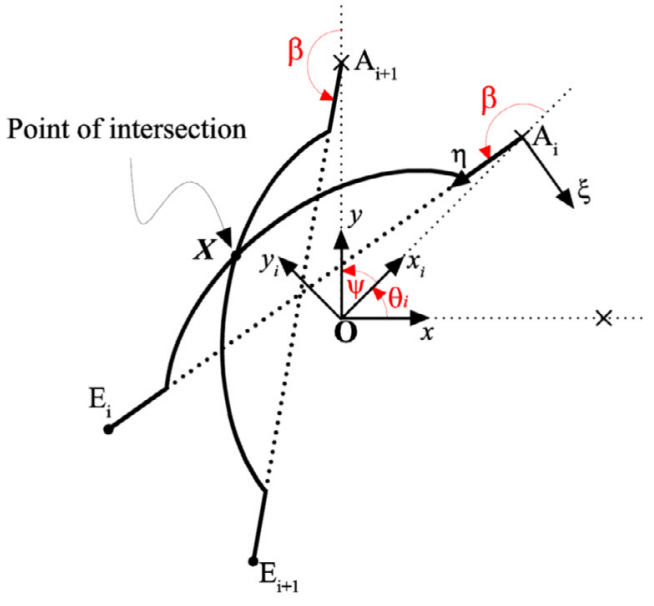

Fig. 11. Geometric relations for two adjacent blades separated by an angle $\psi$ subtended at the center $O$. 


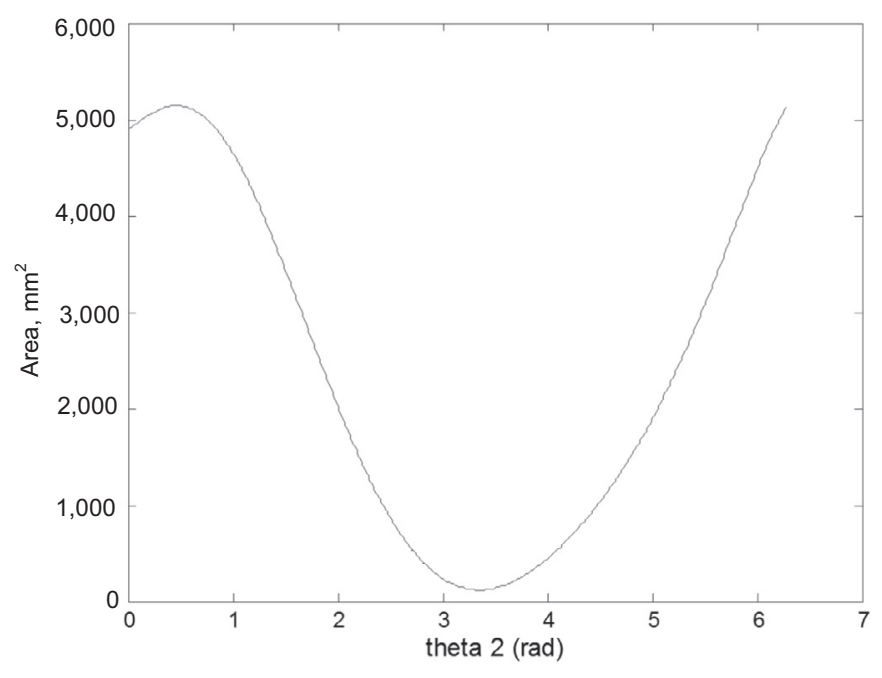

Fig. 12. Variation of area of aperture with $\theta_{2}$.

in temperatures, and (3) their ability to generate very high forces (of the order of $500 \mathrm{MPa}$ ). These three properties make them an ideal material of choice as actuators for demanding applications. For example, SMAs can be used in thermal management of high heat flux devices [20]. Alternatively, they can be used to enhance thermal performance of heat pipe systems [21], whereas they can be used in making mechanical heat-sensitive device to actuate valve opening for achieving fast response in heat pump water heater [22].

SMA based active devices offer a number of advantages over controller driven stepper motors and other such entities since:

- They are triggered by changes in temperature and powered by heat, both of which are readily available in the solar environment.

- They do not need any external power source, and once properly "trained", they need no external controller. In other words, they are self-regulating.

- They do not suffer environmental degradation and damage. Therefore they can be used in a "fit and forget mode" with very little supervision.

- They can generate large forces, and hence can be used to move objects such as the aperture of a solar reactor with little leverage.

Because it is a major challenge to maintain a solar reactor's temperature constant throughout the day, an SMA controlled mechanism is a very attractive method to optimize the use of variable light as opposed to variety of continuously adjustable mechanisms, such as valves, positioners, and focusing systems. One of the challenges of designing such controlled actuators is the fact that solar energy harvesting reactors are installed in remote locations and harsh environments. Solar energy is concentrated to temperatures of $1000{ }^{\circ} \mathrm{C}$ and above via secondary concentrators. Therefore, SMA actuators can be an ideal choice for such conditions.

Identification of the most suitable SMA type for this application was made based on our previous experience on a number of different ideas for SMA based actuators. These ideas include based devices for sun tracking [23], SMA based bending and expansion devices with smart bias [24], and an SMA based device for operating automatic window blinds without motors, sensors, or controllers [25].

While the current actuator is restricted to shape memory springs with actuation temperatures in the vicinity of $100^{\circ} \mathrm{C}$, commercial NiTiPt wires with actuation temperatures of $350^{\circ} \mathrm{C}$ have been developed. These alloys can operate at temperatures of $1000{ }^{\circ} \mathrm{C}$ or higher depending on the weight percentage of Pt. Thus it is potentially possible to use these in a solar reactor's aperture control as well.

\section{Use of SMA spring to control aperture rotation}

Because SMAs have the ability to return to a previously defined shape or size upon application of certain thermal procedure, they are perfect materials to control a solar reactor's aperture opening and closing. There are two types of SMAs: one-way shape memory and two-way shape memory. One-way SMAs exhibit shape memory only when heated, while two-way SMAs demonstrate shape memory when heated and when re-cooled. In this study, a nickel-titanium one-way SMA is used.

SMAs' phase change from martensite to austenite during heating occurs over a range of temperatures. The austenite phase starts to form when the SMA's temperature is raised up to the austenite start temperature, $A_{s}$. The transformation to austenite completes when the temperature reaches the austenite finish temperature, $A_{f}$. Therefore, $A_{f}$ is always greater that $A_{s}$. The difference between the temperatures at which a material is $50 \%$ austenite upon heating and 50\% martensite upon recooling is called hysteresis. A higher hysteresis value indicates a gradually change from martensite to austenite, or from plastic nature to highly elastic state and vice versa.

Two SMA springs were used along with two regular extension springs to bias the SMA springs. The springs were connected to the aperture as shown below in Fig. 13. When the SMA springs are heated, their structure changes to the highly elastic austenite form overcoming the stiffness of the bias springs and rotating the aperture's flange. When the SMA springs cool down, their structure begins to transform from austenite to martensite. Thus, their stiffness reduces. When the stiffness value is below than that of the bias springs, the aperture rotates in the clockwise direction.

Fig. 14 shows the arrangement of the springs with the opening and closing directions with respect to each spring type. The relations of the torque and aperture angle for the SMA and bias springs are provided later. Fig. 15 shows the angular relation between the SMA and the bias spring along with the geometric distances which relate the positions of the springs.

One of the challenges in designing SMA actuators with bias springs is the fact that the bias springs induce a variable force on the SMA springs, thus limiting its stroke. In order to overcome this problem, the bias springs were positioned in such a way that the net torque due to the bias spring is almost constant. This was achieved by mounting the bias springs in such a way that as the spring extends and its tension increases, the angle between the

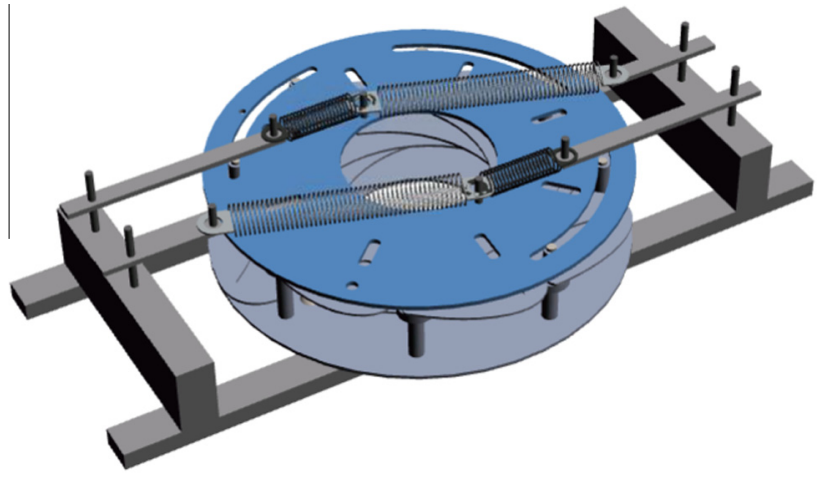

Fig. 13. Experimental setup to control aperture opening and closing with SMA springs [19]. 


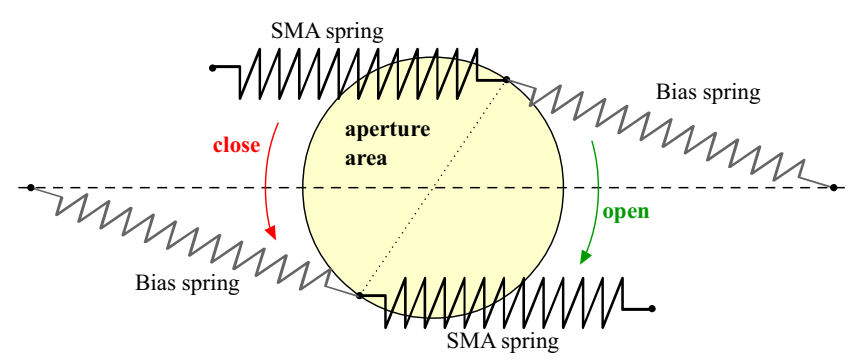

Fig. 14. SMA and bias springs for opening and closing of the aperture area [19].

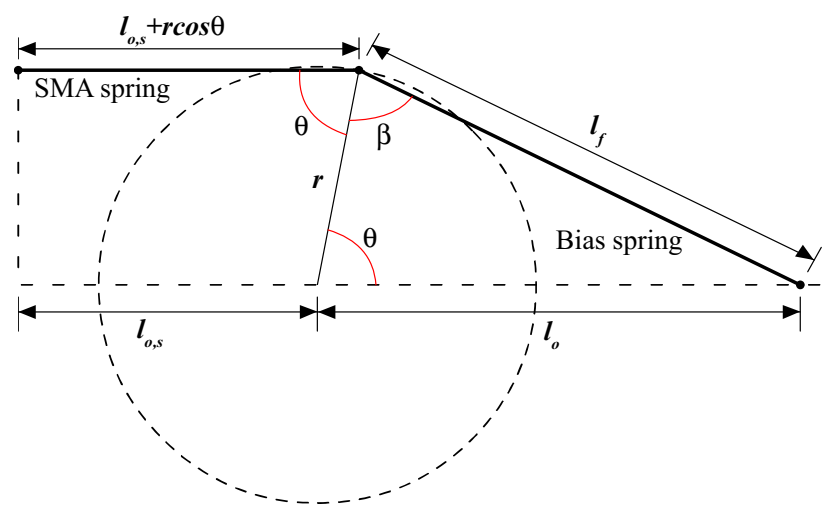

Fig. 15. Arrangement of SMA spring w.r.t. the bias spring on aperture [19].

spring and the radius of the rotating aperture decreases. Therefore, partially compensating for the change in the angle and providing an approximately constant opening torque irrespective of the tension in the springs. The relation of the torque versus angle for the bias spring is as follows:

$\tau=r \cdot F \sin \beta$

$\tau(\theta)=r k\left[1-\frac{l_{i}}{l_{f}(\theta)}\right] l_{o} \sin \theta$

$l_{f}(\theta)=\sqrt{l_{o}^{2}+r^{2}-2 r l_{o} \cos \theta}$

In deriving the above equation, we have set the free length of the bias spring to be $l_{i}$ and have used the sine rule and the cosine rule. For the spring used, Eqs. (28) and (29) can be plotted to show the bias spring's torque as a function of the aperture rotation. This is shown in Fig. 16.

The torque of the SMA spring is a function of temperature and aperture rotation angle, $\tau_{s}=f(T, \theta)$. In order to quantify the variation of stiffness of the SMA spring with temperature, a series of tests on the SMA springs were conducted with different dead loads and at the $A_{s}$ and $A_{f}$ temperatures. Based on this, a linear variation of the SMA stiffness with temperature was assumed. The results are in accord with what is used in conventional SMA design [26]. Based on the geometry shown in Fig. 15, we can derive the torque produced by the SMA spring as a function of the temperature and rotation angle as follows:

$\tau_{s}=r \cdot F_{s} \sin \theta$

$k(T)=k_{m} T+k_{o}$

$\tau_{s}=f(T, \theta)=r\left(k_{m} T+k_{o}\right)\left(l_{o, s}+r \cos \theta-l_{i, s}\right) \sin \theta$

In above equations, $k(T)$ is the temperature dependent stiffness of the SMA spring and $l_{i, s}$ is the initial (coil bound) length of the spring.

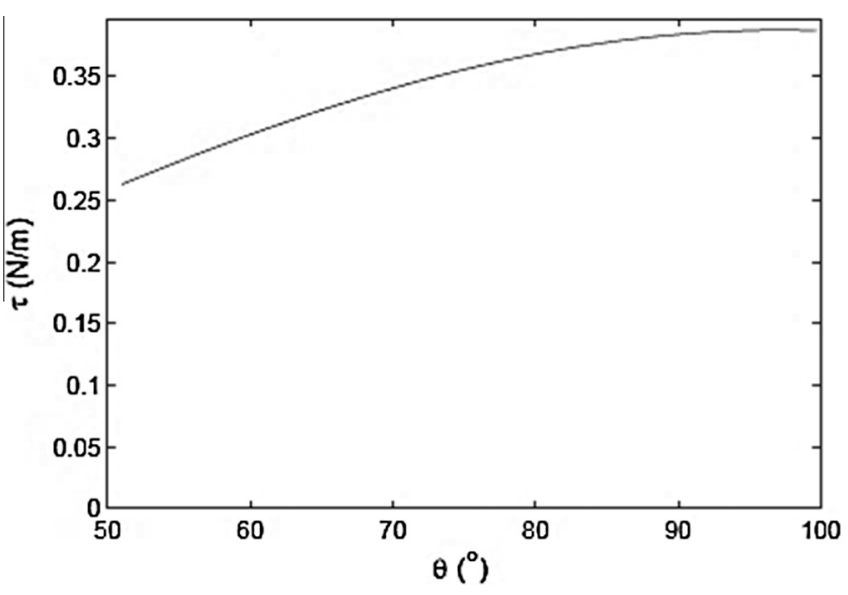

Fig. 16. Bias spring torque variation with aperture rotation angle $\theta$.

\section{Experiments and results}

Feasibility of using SMA springs to open and close the iris aperture mechanism was tested in two methods. The first method was conducted through electric heating, while the second method was conducted with the use of a solar simulator as the heat source. The first set of experiments was performed with ten SMA springs for the following tests:

(1) Timing the opening/closing of the aperture with SMAs.

(2) Determination of SMA's behavior at low and high temperatures with respect to its force deflection curve.

For the first test, two SMA springs were connected in series to a $2.5 \mathrm{~V}$ DC power supply. Relatively high electrical resistance of the SMA resulted in electrical heating of the SMA and therefore initiated its phase transformation. Once the aperture's radius reached the lower limit $\left(r_{\text {close }}\right)$, the power supply was switched off and the SMA was allowed to cool down. When the SMA's temperature was decreasing, the bias spring stiffness took over and the aperture area started expanding. Duration of the time taken for the aperture to open and close between two fixed radii was noted for each pair of SMA springs. The average aperture's opening and closing radii considered during time measurement were: $r_{\text {open }}=36.59 \mathrm{~mm}$ and $r_{\text {close }}=26.37 \mathrm{~mm}$. For the second test, an SMA spring, in its austenite size and shape but in the martensite form at room temperature, was loaded with a known mass and the corresponding deflection was noted. The SMA spring was then heated so that it returns to its memorized shape. Then it was allowed to cool in order to return to martensite phase. The above procedure was repeated for each of the ten SMA springs.

Fig. 17 shows the results for opening and closing the aperture for the five pairs of SMA springs. Except for SMA pair 5, the rest of the pairs took significantly less time to close the aperture than to open it. For instance, $t_{\text {close.avg }} \approx 20 \mathrm{~s}$, while $t_{\text {open.avg }} \approx 88 \mathrm{~s}$. This was due to the relatively high rate of heating caused by the continuous supply of electrical power until $r_{\text {close }}$ was reached. It takes more time for the bias springs to open the aperture as a result of the slow rate of cooling of SMAs by natural convection. The response time for opening and closing the aperture is significantly less than the time scales involved with daily variations in solar irradiation due to weather changes.

The force-deflection of the SMA springs is plotted in Fig. 18 along with the spread in deflection $(\delta x)$ for each loading. The mean value for the SMA spring constant at the high temperature was $k_{H}=249 \mathrm{~N} / \mathrm{m}$, whereas it was $k_{L}=102 \mathrm{~N} / \mathrm{m}$ at the low 

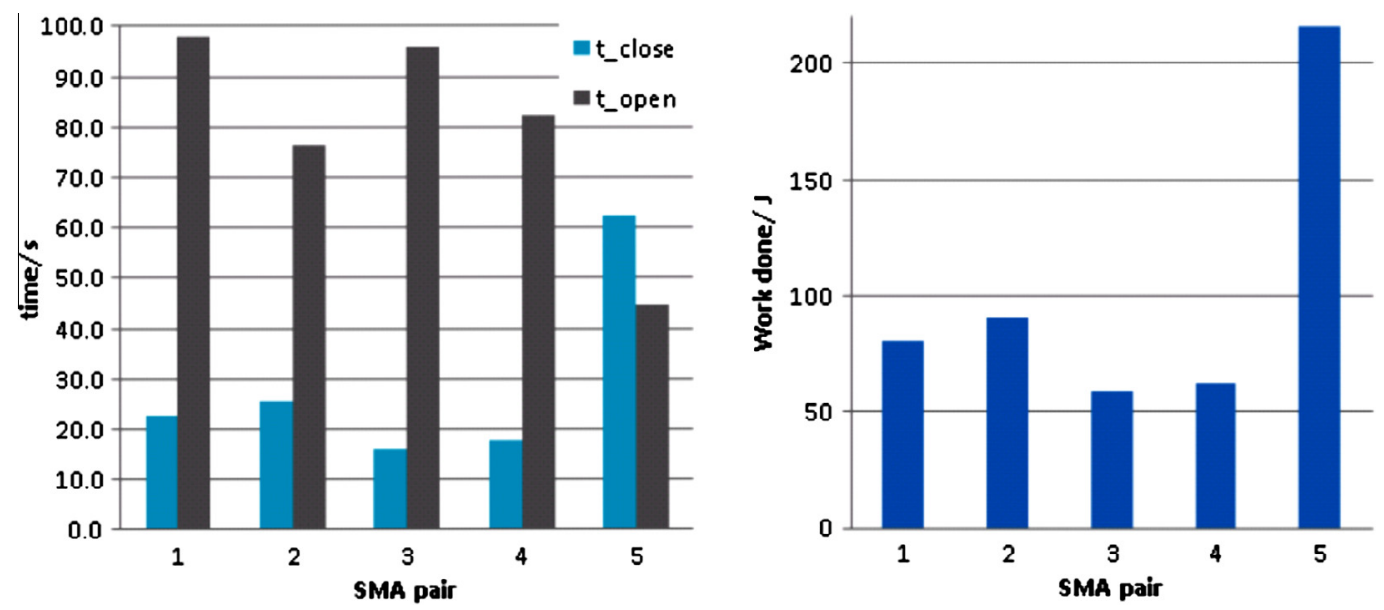

Fig. 17. Experimental results for different SMA spring pairs [19].

temperature. Since the transformation from martensite to austenite begins at $A_{s}$ and reaches completion at $A_{f}, k_{H}$ and $k_{L}$ were assumed to correspond to $A_{f}$ and $A_{s}$. Given that $A_{f}$ and $A_{s}$ values are known from the manufacturer database, Eq. (31) gives that $k_{m}=7.38 \mathrm{~N} / \mathrm{m}$ and $k_{o}=-2320 \mathrm{~N} / \mathrm{m}$.

For static equilibrium of the aperture, $\tau(q)=\tau_{s}(T, \theta)$. Therefore, Eqs. (28) and (30) were solved for the equilibrium temperature and rotation angle. The result is plotted in Fig. 19, where there is one equilibrium temperature, $T_{\text {eqm }}$, for a particular $\theta$. If the SMA is at $T_{\text {eqm }}$, then the system is in static equilibrium. Thus, for the SMA spring to dominate over the regular spring, the SMA temperature must be above the $T_{e q m}-\theta$ curve shown in Fig. 19. On the other hand, for temperatures below $T_{\text {eqm }}$, the regular spring will bias the SMA spring and the aperture will open.

The second set of experiments was done with a $7 \mathrm{~kW}$ solar simulator. Details of the solar simulator can be found in a previous publication [27]. In order to quantify the variation of force exerted by an SMA spring with respect to temperature, the experiment shown in Fig. 20 was arranged. A tension SMA spring was used for this experiment. Fig. 20 shows a schematic of the experimental setup and a cross section of the spring containment unit placed in the Erlenmeyer flask. The purpose of this experiment was to get an estimate of the range of forces the SMA springs could exert and their corresponding range of temperature.

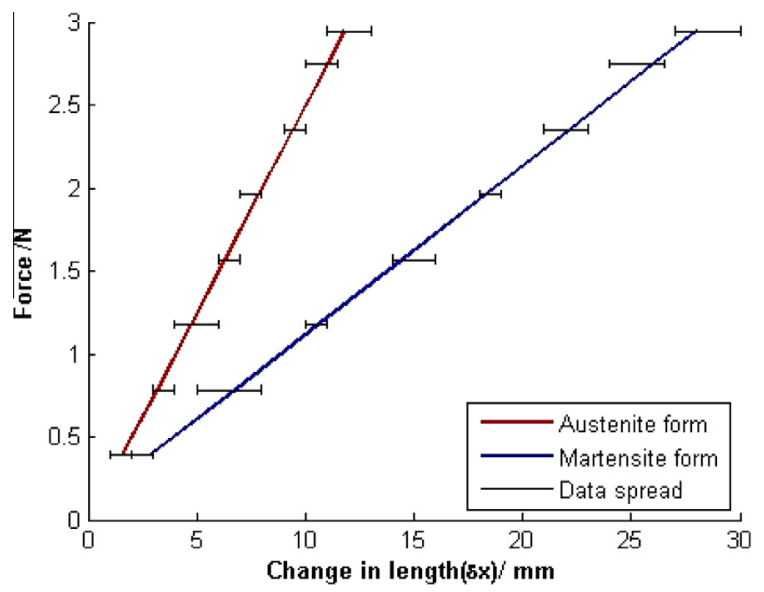

Fig. 18. Force-deflection curves at temperatures below $A_{s}$ and above $A_{f}$ along with variation in $d x$ for each loading [19].

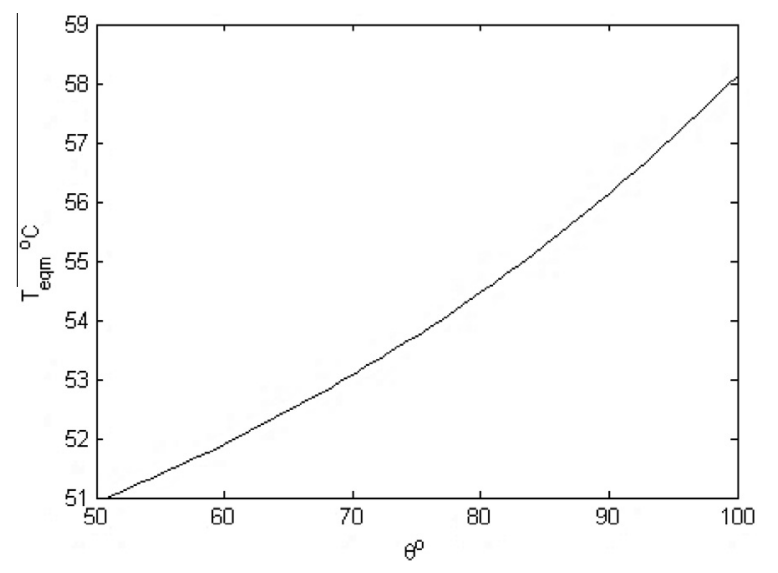

Fig. 19. SMA temperature at equilibrium and corresponding aperture rotation angle $\theta$.

Prior to the experiment, the spring was stretched beyond its elastic limit at room temperature using a $400 \mathrm{~g}$ weight. The temperature was then increased by heating the hot plate and the force exerted by the spring was measured using a force sensor. The hot plate provided a specified constant heat flux to the beaker. Realtime variations of temperature and spring force were recorded and the results are plotted below. Fig. 21 demonstrates the time response of the force exerted by the SMA spring as a result of the heating, while Fig. 22 illustrates the variation of the force with temperature.

Results in Fig. 21 show that the time response of the actuation was relatively small when compared to the time involved in variations of solar radiation. Therefore, demonstrating that the time response of SMA springs should cause an insignificant impact on the operation of the solar reactor's aperture. The results also validate the principle that the solid-state phase change in SMAs is not dependent on time, as most other phase changes, but rather is only dependent on temperature. On the other hand, results in Fig. 22 demonstrate how the change in force is increasing with the increase in temperature. Thus, suggesting that the stiffness of the SMA spring is also increasing with temperature. The maximum recorded force was around $1.3 \mathrm{~N}$, which was the force at which the spring has approached its initial state (before being plastically deformed). However, if that was not the case, the force would be expected to keep increasing with temperature in a non-linear manner as that exhibited at lower temperatures. 

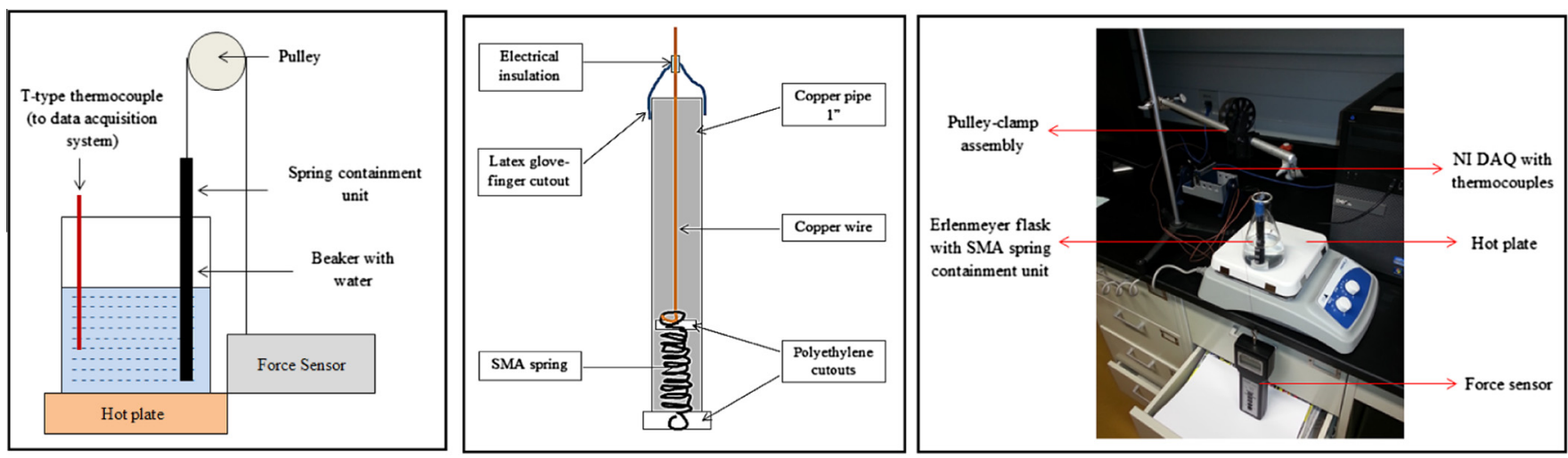

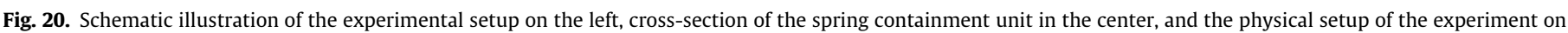
the right [19].

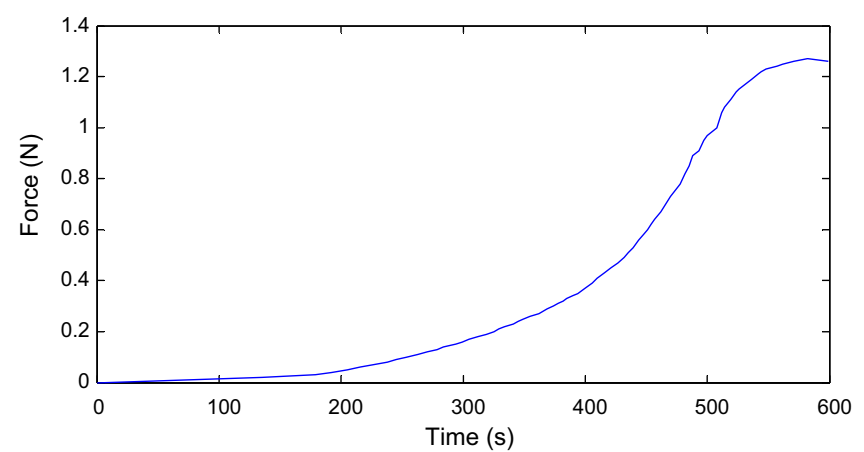

Fig. 21. Variation of force exerted by spring with time.

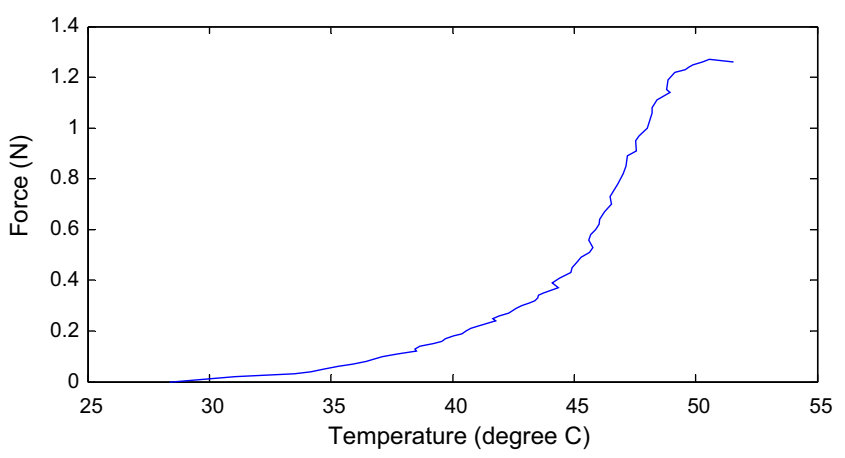

Fig. 22. Variation of force exerted by spring with temperature of the spring.

\subsection{Uncertainty analysis}

The results of the second set of experiments, either in Figs. 21 and 22 above or Figs. 24-26 below, had some errors. These errors were required to be analyzed to ensure that the results obtained and any observations made are viable. The method used for the uncertainty analysis was acquired from the American National Standards Institute (ANSI) [28]. This method was based on separating the uncertainty into two types; type A and type B. Type A was due to the experimental setup and the effect it had on the measurements, while type B was due to uncertainty in the sensors (force sensor). Eq. (33) below was used in order to calculate the total uncertainty, with a $95 \%$ confidence level.

$U_{\text {Total }}= \pm 2\left[\left(U_{A}\right)^{2}+\left(U_{B}\right)^{2}\right]^{\frac{1}{2}}$

where type A and type B uncertainties corresponds to $U_{A}$ and $U_{B}$, respectively.

The uncertainty of the experimental setup was $\pm 0.03 \mathrm{~N}$ due to fluctuation in the readings observed during the experiments, while the uncertainty of the force sensor was $0.01 \mathrm{~N}$ as provided by its manufacturer. Therefore, the total uncertainty was calculated and found to be $\pm 0.06 \mathrm{~N}$ with a $95 \%$ confidence level. This corresponds to approximately $5 \%$ relative error.

After completing this step, the same experiment was repeated using the solar simulator instead of the hot plate. The experimental setup was shifted to the front of the simulator as shown in Fig. 23.

This experiment was conducted three times for varying levels of heating by the solar simulator. The three levels, which were at $110 \mathrm{~A}, 125 \mathrm{~A}$ and $153 \mathrm{~A}$, are representative of varying solar fluxes that are intercepted on the earth at different times during the
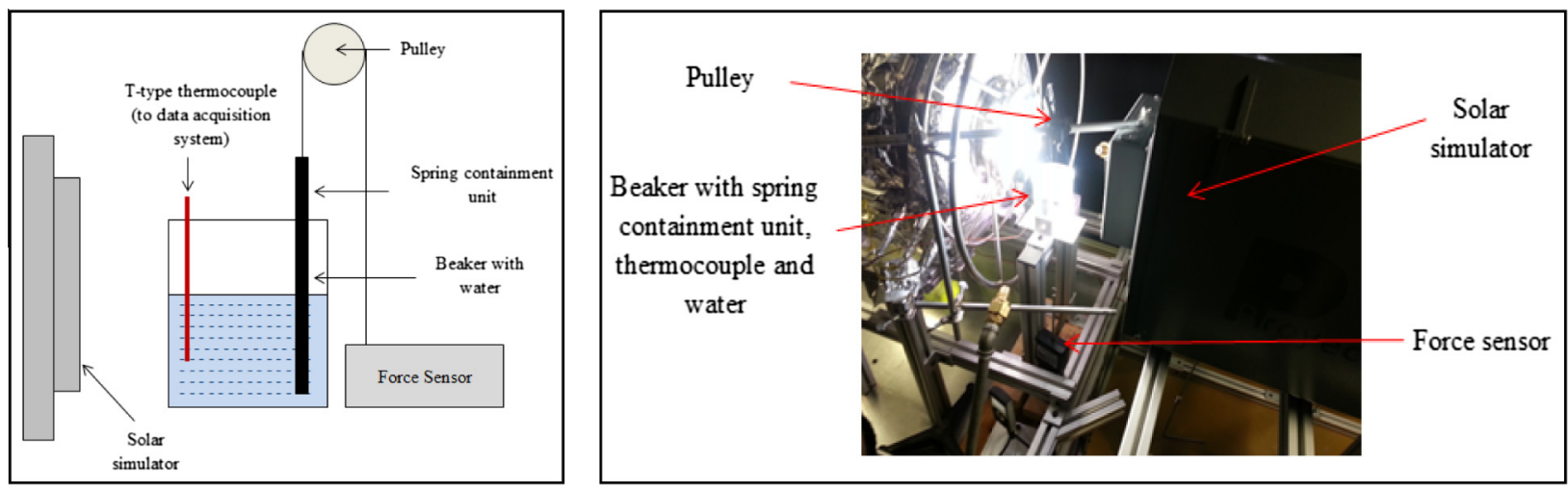

Fig. 23. Schematic of the setup on the left and the physical setup of the experiment to test SMA spring with solar simulator as the heating source.

Please cite this article in press as: A. Rajan et al., An experimental and analytical study on the feasibility of SMA spring driven actuation of an iris mechanism, Appl. Therm. Eng. (2016), http://dx.doi.org/10.1016/j.applthermaleng.2016.04.033 


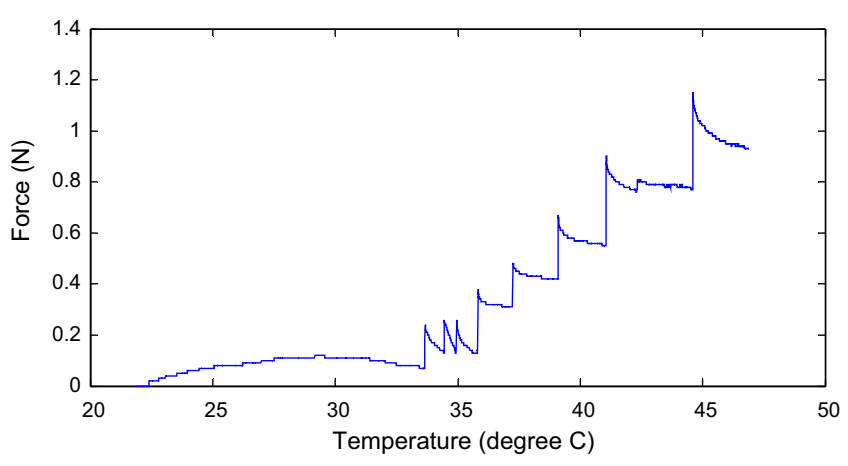

Fig. 24. Variation of SMA spring force with temperature for simulator at $110 \mathrm{~A}$.

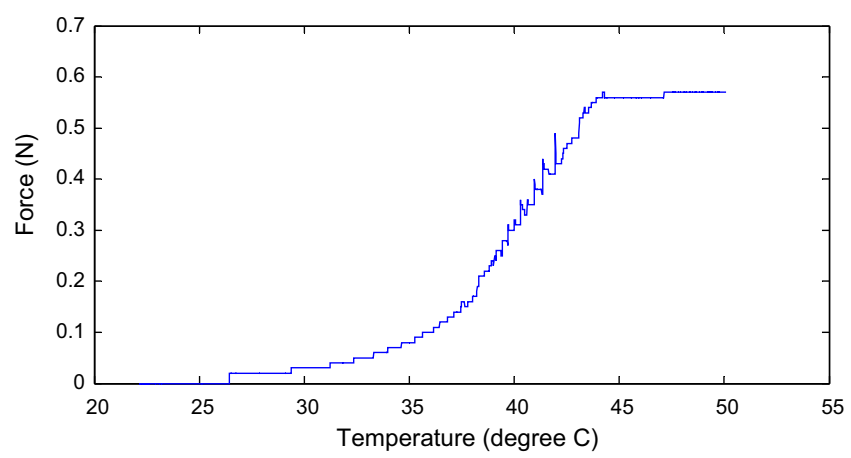

Fig. 25. Variation of SMA spring force with temperature for simulator at $125 \mathrm{~A}$.

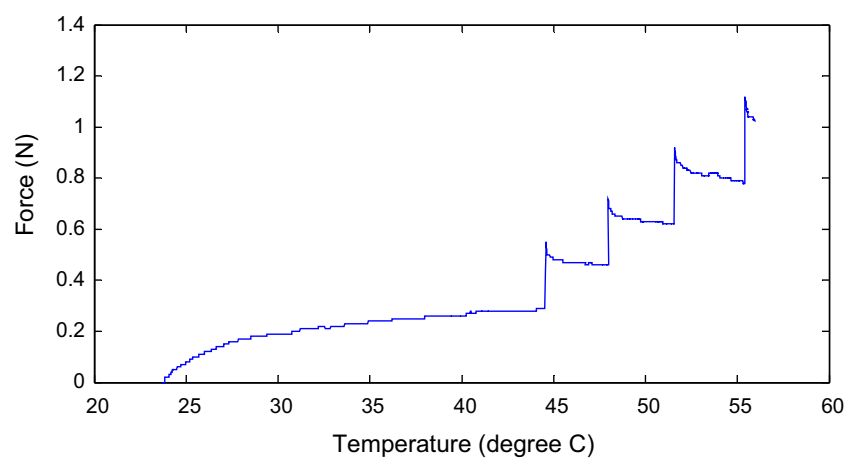

Fig. 26. Variation of SMA spring force with temperature for simulator at $153 \mathrm{~A}$. day (sunrise to sunset). Figs. 24-26 below show the variation of the SMA spring force with respect to temperatures. The error associated with the results in the figures below is the same as the error calculated earlier for Figs. 21 and 22, since the experimental setup and sensors used were the same.

It can be observed from Figs. 24-26 that the variation of force with increasing temperature for the SMA spring is non-linear. The spring exerts greater force with increasing temperature. As the temperature increases, the stiffness of the spring increases. At high enough temperatures, the spring returns to its original state prior to its extension beyond its elastic limit.

By examining the results in Figs. 21, 22, 24-26, it can be noticed that all of them exhibit roughly the same behavior. The force exerted by the SMA spring in all cases starts to increase slowly with temperature, and then suddenly increases rapidly. This is due to the fact that as the temperature increases, it hits the temperature at which the transition from the martensite phase to the austenite phase starts occurring. Hence, the force starts increasing at a much larger rate.

As discussed earlier, SMA springs have the ability to memorize their shape and the corresponding spring constant at different temperatures. The aperture mechanism uses linkages to transmit motion generated by a motor to the top plate and blades. All experiments presented above prove that by employing a heating mechanism for an SMA spring, it will be possible to replace the linkages with these springs and discard the use of any motors. Fig. 27 below shows how the bar linkage in the current aperture design may be replaced by an SMA spring.

\section{Conclusions}

The goal of this paper was to evaluate the possibility of using SMA springs to actuate a variable aperture mechanism that helps maintain the efficiency of a solar reactor.

Theoretical and experimental proof-of-concept of an SMA controlled variable aperture mechanism was presented. It was observed that when the SMA springs were heated, their structure changed from the plastic martensite form to the highly elastic austenite form. Therefore, overcoming the stiffness of the bias springs and rotating the aperture's flange. However, the variation of force with increasing temperature for the SMA spring was found to be non-linear. As the temperature increases, the stiffness of the spring increases. On the other hand, the time response of the actuation was observed to be relatively small when compared to the time involved in variations of solar radiation. It was also observed that the time for closing the aperture was much less that the time for opening the aperture, where $t_{\text {close, avg }} \approx 20 \mathrm{Xs}$, while $t_{\text {open,avg }} \approx 88 \mathrm{~s}$. This is an asymmetric response, where the actuation time is fast while the de-actuation time is slow. In order to enhance
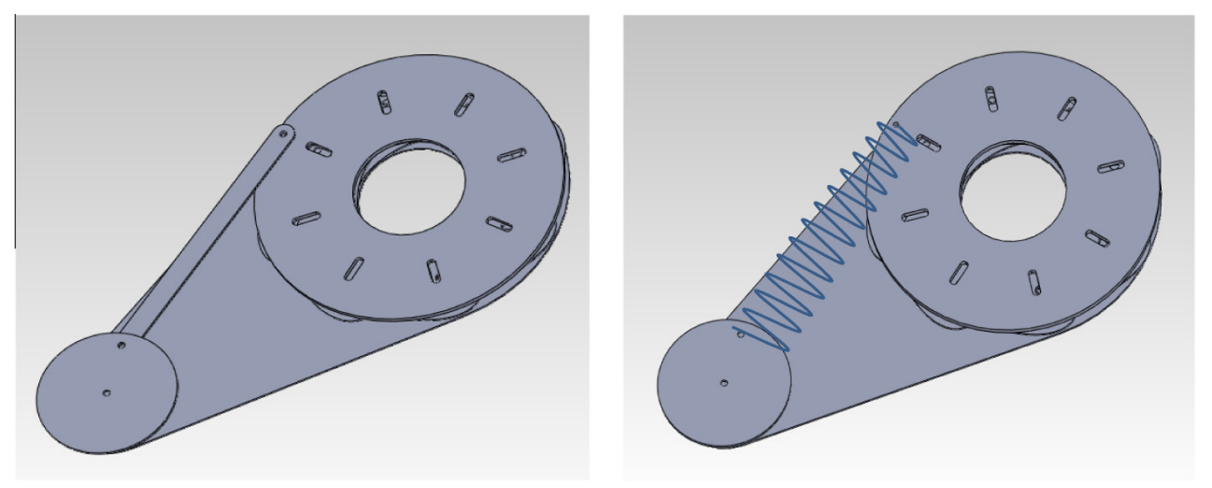

Fig. 27. Bar linkage (featured in the aperture design on the left) is replaced by SMA spring in the aperture on the right. 
this response, ways of increasing the heat transfer from the SMA to the surrounding environment may be induced.

All experiments presented above showed that by employing a heating mechanism for SMA springs, it is possible to replace the linkages with these springs and discard the use of any motors. Using SMA springs instead of motors will not only save energy, but will also simplify the control setup. Furthermore, the current design of the aperture mechanism puts the motor close to the solar simulator where temperatures can get extremely high. This could result in great damage to the motor involved.

To sum up, the actuation for opening and closing the iris mechanism can be provided by the aperture temperature and no external source of actuation would be required. The experiments performed are a preliminary study to explore the range of forces available and the corresponding temperatures, as far as the SMA springs are concerned. The studies have shown that using SMA springs to control the variable aperture looks very plausible and beneficial.

With further design iterations, this concept can be fine-tuned to open and close the aperture precisely as required. Therefore, unique character of SMAs in sensing the variations of heat flux can be a promising alternative to traditional temperature sensors and the controllers in solar thermal applications. With little more future work, there is a good possibility that SMA springs may be able to replace the motor and linkages in the current aperture mechanism design. Future work on this subject revolves around introducing a heating resistor and developing a control mechanism to regulate the current to the resistor. Future studies may also include experimenting the effect of fatigue on the function of SMAs and how their memory would be affected by time.

The following are few vistas by which the accuracy of the experiments could have been improved.

- Use a clamp to hold the SMA assembly down.

- Use a force sensor that can provide real-time force readings with LabVIEW.

- Compare runs with a varying prior extensions.

\section{Acknowledgments}

This publication was made possible by the seed fund from the Office of Research and Graduate Studies (RGS) of Texas A\&M University at Qatar, and NPRP grant 09-670-2-254 from the Qatar National Research Fund.

\section{References}

[1] M. Roeb, C. Sattler, Isothermal water splitting, Science 341 (2013) 470-471.

[2] J.P. Säck, M. Roeb, C. Sattler, R. Pitz-Paal, A. Heinzel, Development of a system model for a hydrogen production process on a solar tower, Sol. Energy 86 (2011) 99-111.

[3] J. Corey, M.T. Noone, A. Mitsos, Heliostat field optimization: a new computationally efficient model and biomimetic layout, Sol. Energy 86 (2) (2012) 792-803.
[4] N. Ozalp, D. Jayakrishna, Numerical study on the thermal interaction of gasparticle transport for a vortex flow solar reactor, in: ASME 4th International Conference on Energy Sustainability (ES2010), Paper No: ES2010-90325, 2010.

[5] S. Abanades, G. Flamant, Production of hydrogen by thermal methane splitting in a nozzle-type laboratory-scale solar reactor, Int. J. Hydrogen Energy 30 (2005) 843-853.

[6] V. Shilapuram, D. JayaKrishna, N. Ozalp, Residence time distribution analysis of aero-shielded solar cyclone reactor for emission-free generation of hydrogen, Int. J. Hydrogen Energy 36 (2011) 13488-13500.

[7] M. Chien, N. Ozalp, G. Morrison, CFD and heat transfer analysis of vortex formation in a solar reactor, ASME J. Therm. Sci. Eng. Appl. 7 (2015) $0410071-$ 0410078.

[8] N. Ozalp, A. Toyama, D. Jayakrishna, R. Rowshan, Y. Al-Hamidi, Effect of camera-like aperture in quest for maintaining quasi-constant radiation inside a solar reactor, ASME J. Mech. Des. 133 (2) (2011) 021002.

[9] S. Usman, N. Ozalp, Numerical and optical analysis of solar power level adaptable solar reactor, Heat Transfer Eng. 35 (16) (2014) 1405-1417.

[10] NREL Database Retrieved in March 2016 at <www.nrel.gov/midc/srrl_rsp2>.

[11] R.E. Bird, R.L. Hulstrom, Simplified Clear Sky Model for Direct and Diffuse Insolation on Horizontal Surfaces, Technical Report No. SERI/TR-642-761, Golden, CO, 1981.

[12] A. Steinfeld, M. Schubnell, Optimum aperture size and operating temperature of a solar cavity-receiver, Sol. Energy 50 (1993) 19-25.

[13] L. Ren, S. Park, H. Ren, I. Yoo, Adaptive liquid lens by changing aperture, J. Microelectromech. Syst. 21 (4) (2012) 953-958.

[14] R. Syms, H. Zou, J. Stagg, H. Veladi, Sliding-blade MEMS iris and variable optical attenuator, J. Micromech. Microeng. 14 (2004) 1700-1710.

[15] E. Graves, H. Zhou, R. Chatterjee, P. Keall, S. Gambhir, C. Contag, A. Boyer, Design and evaluation of a variable aperture collimator for conformal radiotherapy of small animals using a microCT scanner, Med. Phys. 34 (11) (2007) 4359-4367.

[16] R. Hill, Variable Aperture Collimator for High Energy Radiation, U.S. Patent No. 4,450,578, 1984.

[17] G. Chang, J. Peng, C. Chan, Multi-Blade Lens-Aperture Control Mechanism, U.S. Patent No. 6,161,966, 2000.

[18] G. Echner, W. Kilby, M. Lee, E. Earnst, S. Sayeh, A. Schlaefer, B. Rhein, J. Dooley, C. Lang, O. Blanck, E. Lessard, C. Maurer, W. Schlegel, The design, physical properties and clinical utility of an iris collimator for robotic radiosurgery, Phys. Med. Biol. 54 (2009) 5359-5380.

[19] A. Rajan, M. Abouseada, P. Manghaipathy, A. Srinivasa, N. Ozalp, Feasibility of using shape memory alloy (SMA) spring to facilitate actuation of a variable aperture mechanism of a solar reactor, Chem. Eng. Trans. 45 (2015) 841-846.

[20] A.M. Waddell, J. Punch, J. Stafford, N. Jeffers, On the hydrodynamic characterization of a passive shape memory alloy valve, Appl. Therm. Eng. 75 (2015) $731-737$.

[21] M.S. Aris, R. McGlen, I. Owen, C.J. Sutcliffe, An experimental investigation into the deployment of 3-D, finned wing and shape memory alloy vortex generators in a forced air convection heat pipe fin stack, Appl. Therm. Eng. 31 (2011) 2230-2240.

[22] B.J. Huang, J.H. Wang, J.H. Wu, P.E. Yang, A fast response heat pump water heater using thermostat made from shape memory alloy, Appl. Therm. Eng. 29 (2009) 56-63.

[23] N. Ganesh, S. Maniprakash, L. Chandrasekaran, S. Sivakumar, A.R. Srinivasa, Design and development of a sun tracking mechanism using the direct SMA actuation, ASME J. Mech. Des. 133 (7) (2011) 075001-075008.

[24] A. Rao, P. Ghosh, A.R. Srinivasa, Design of multi-state and smart-bias components using shape memory alloy and shape memory polymer composites, J. Mater. Des. 44 (2013) 164-171.

[25] S.M. Sivakumar, V. Buravalla, A.R. Srinivasa, Smart Devices: Modeling of Material Systems, American Institute of Physics, 2010. ISBN-10: 0735405530.

[26] T. Warram, Actuator Design Using Shape Memory Alloys, Hamilton, Ontario, Canada, 1993.

[27] J. Sarwar, G. Georgakis, R. LaChance, N. Ozalp, Description and characterization of adjustable flux solar simulator for solar thermal, thermochemical and photovoltaic applications, Sol. Energy 100 (2014) 179-194.

[28] American National Standards Institute (ANSI), American National Standard for Expressing Uncertainty - U.S. Guide to the Expression of Uncertainty in Measurement, ANSI/NCSL Z540-2-1997, 1997. 